\title{
Experiences with simultaneous use of contraception and the vaginal ring for HIV prevention in sub-Saharan Africa
}

\author{
Jonah Leslie ${ }^{1 *}$ (D), Flavia Kiweewa ${ }^{2}$, Thesla Palanee-Phillips ${ }^{3}$, Katherine Bunge ${ }^{4}$, Felix Mhlanga ${ }^{5}$, Betty Kamira ${ }^{2}$, \\ Jared Baeten ${ }^{6}$, Ariana Katz ${ }^{1}$, Sharon Hillier ${ }^{7}$ and Elizabeth Montgomery ${ }^{1}$ for the MTN-020/ASPIRE Study Team
}

\begin{abstract}
Background: Clinical trials have found that a monthly dapivirine vaginal ring was well-tolerated and reduced HIV-1 risk among women in sub-Saharan Africa. However, in order for the ring or other novel prevention methods to have optimal impact, it is necessary to understand and address women's challenges to uptake and adherence. This paper provides insight into a few key challenges noted by women using the ring and contraceptives simultaneously.

Methods: The qualitative portion of the MTN-020/ASPIRE study consisted of data collection using single in-depth interviews, serial in-depth interviews, and focus group discussions, conducted with 214 participants across 15 sites in Malawi, South Africa, Uganda and Zimbabwe. A coding team used qualitative analysis software to identify themes within the interviews.

Results: The primary qualitative themes among participant data pertained to side effects. Participants reported negative side effects related to menses, in some cases attributing these effects to their contraceptives and in others to the vaginal ring. Participants also expressed concern over the long-term impact of contraception and ring use on fertility, including the reversibility of the contraceptive, especially among nulliparous women.

Conclusions: Women's attitudes toward contraceptives can impact their willingness to concurrently use and adhere to a novel HIV prevention product. To optimize the potential of both prevention products, researchers should preemptively address concerns about contraceptive impact on fertility and counsel women about the expected side effects of contraceptives versus the ring.

Clinical trials identifier NCT01617096. Registered on 6-12-2012 at clinicaltrials.gov https://clinicaltrials.gov/ct2/show/ NCT01617096
\end{abstract}

Keywords: HIV, Contraception, Qualitative research, Vaginal ring, Africa South of the Sahara, Clinical trial

\section{Background}

Women of childbearing age in sub-Saharan Africa continue to face disproportionate risk of HIV-1 acquisition compared to men and their global counterparts [1]. Two

\footnotetext{
*Correspondence: jleslie.anthro@gmail.com

${ }^{1}$ Women's Global Health Imperative, RTI International, San Francisco, CA, USA

Full list of author information is available at the end of the article
}

clinical trials (MTN-020/ASPIRE and IPM 027/The Ring Study) reported results showing that a monthly dapivirine vaginal ring was well-tolerated and reduced HIV-1 risk among women in sub-Saharan Africa [2, 3]. Open label extension studies of the vaginal ring have recently completed, confirming the safety profile of the ring as well as demonstrating a reduction in expected HIV-1 incidence [4]. 
As was demonstrated in the Phase III and open label extension studies, the efficacy of the ring relies on its consistent use. In the MTN 020/ASPIRE trial, women were required to use a highly effective method of contraception throughout their participation, as the effect of the drug and ring on the fetus or pregnancy was unestablished [2]. In order for the ring or other novel prevention methods to have optimal impact on reducing risk of HIV infection, it is necessary to address factors that influence women's fears and concerns [5], as well as other challenges to uptake and adherence. Concurrent use of an HIV prevention product and a contraceptive is one such challenge. Widespread adoption of the dapivirine ring requires that issues around users' fear of contraceptive side effects be recognized in the context of adherence to the ring.

MTN020/ASPIRE participants were provided with a choice of contraceptive pills, injections (DMPA, Net-En), implants (Jadelle, Nexplanon), or the copper IUD, either directly at the trial sites or through referrals within the public sector. They were also offered contraceptive counseling. This qualitative methods paper provides insight into some of the key challenges noted by users of the ring and contraceptives, and addresses implications of these challenges for the future success of the dapivirine ring.

\section{Methods}

\section{Population and study design}

In the full ASPIRE trial, a total of 2629 women were enrolled across 15 sites in Malawi, South Africa, Uganda, and Zimbabwe [2]. The ASPIRE study design, population and procedures are published elsewhere, but briefly, women were aged 18-45 and were HIVnegative, nonpregnant or breastfeeding and sexually-active at enrollment [2]. They were followed for a minimum of 12 months from 2012 to 2015, with a qualitative component conducted at six sites spanning from 2013 to 2015 [6]. The qualitative portion consisted of single in-depth interviews (IDI), serial IDIs (SIDI), and focus group discussions (FGD), conducted with a total of 214 participants composed of those who were randomly preselected from ASPIRE participants, those who were purposively selected as "special cases" based on their unique adherence experiences, and those who discontinued early because of HIV seroconversion [6]. SIDI allowed for the study of longitudinal themes and increased rapport between interviewers and respondents. IDI targeted trial participants who had discontinued ring use, typically due to seroconversion, pregnancy, or grade 3 or 4 adverse events. FGD were conducted to examine group perceptions of the ring. The SIDI and IDI interview guide is provided as Additional File 1. The FGD interview guide is provided as Additional File 2. SIDI were conducted near the conclusion of participants' third and twelfth months in the study, and around the time of their Product Use End Visit (PUEV). FGD were conducted at or around participants' PUEVs. The timing of single IDI varied.

Modern contraception was a requirement for trial eligibility. The study included interviews with participants who initiated or changed contraception methods prior to enrollment, as well as those already using effective methods. Contraceptive counseling, covering the expected efficacy, use, and mechanism of each method, was offered at screening and enrollment visits, as well as all follow-up visits. Participants were also counselled on potential side effects and ways that these side effects could be clinically managed. Counselling was typically provided by nurses, with support by physicians and pharmacists. When experiencing side effects, participants were provided with medication or guidance on changing methods, which were provided at no cost.

\section{Data collection and analysis}

Sixteen Institutional or Ethics Review Boards, representing each qualitative data collection site approved the study. All participants provided written informed consent. Interviews were conducted in local languages by trained social scientists using semi-structured guides covering participants' motivation to join the trial, perceptions of HIV risk, trial experiences, male partner perceptions, and the ring's effect on sex. Participants were further queried about how their participation in ASPIRE influenced their contraceptive use and their experiences using the ring during menses, with probes to explore their experience of side effects. Interviews were audio-recorded, transcribed verbatim, and translated into English before being uploaded into NVivo 11 qualitative analysis software for coding. Both deductive and inductive coding was employed, with checks to maintain $90 \%$ intercoder reliability on $10 \%$ of interviews. The coding team discussed themes over a 14 month period. Themes surrounding contraception concerns were examined by a subset of analysts and coauthors. Specifically, the following codes were analyzed for this paper: Contraception, Discontinuation, Removal, Side Effects, Staff, Negative Influence, and Positive Influence. Because the area of contraceptive challenges was not a key objective of the ASPIRE study, the topics addressed in this paper arose unevenly across participant interviews. Therefore, descriptors of specific quantitative prevalence have been avoided in favor of focusing on the salience of described themes, with an emphasis on exemplary quotes as preferred for qualitative reporting of this nature [7]. 


\section{Results}

Table 1 displays participant characteristics of the qualitative subset of the study population. Participants' ages ranged from 18 to 24 , with a median age of 26 years. $73 \%$ had completed at least some secondary school. 55\% were not married.

The primary qualitative themes about contraceptive use pertained to side effects, both immediate effects on health and comfort, and fears of long-term effects on fertility.

Participants across multiple sites reported experiencing side effects related to menses, including spotting or heavy bleeding, prolonged menses, irregular menstruation, and

Table 1 Participant characteristics

\begin{tabular}{|c|c|}
\hline & QUAL sample \\
\hline Participants enrolled, N & 214 \\
\hline \multicolumn{2}{|l|}{ Currently married } \\
\hline Yes & $96(45 \%)$ \\
\hline No & $118(55 \%)$ \\
\hline \multicolumn{2}{|l|}{ Highest level of education } \\
\hline No schooling & $5(2 \%)$ \\
\hline Primary school (partial and complete) & $45(21 \%)$ \\
\hline Secondary school (partial and complete) & $156(73 \%)$ \\
\hline Attended college or university & $8(4 \%)$ \\
\hline \multicolumn{2}{|l|}{ Primary partner knows of participation in the trial } \\
\hline Yes & $153(72 \%)$ \\
\hline No & $60(28 \%)$ \\
\hline Not sure & - \\
\hline \multicolumn{2}{|l|}{ Primary partner knows has been asked to use ring } \\
\hline Yes & $125(59 \%)$ \\
\hline No & $88(41 \%)$ \\
\hline Not sure & - \\
\hline \multicolumn{2}{|l|}{ Primary partner HIV status } \\
\hline HIV positive & $4(2 \%)$ \\
\hline HIV negative & $125(59 \%)$ \\
\hline Participant does not know & $84(39 \%)$ \\
\hline \multicolumn{2}{|l|}{ Had same primary partner for last 3 months } \\
\hline Yes & $206(97 \%)$ \\
\hline No & $7(3 \%)$ \\
\hline \multicolumn{2}{|l|}{ Current method of contraception } \\
\hline Intrauterine device & $25(12 \%)$ \\
\hline Oral contraceptives & $14(7 \%)$ \\
\hline Injectable contraceptives & $121(57 \%)$ \\
\hline Depot medroxyprogesterone acetate (DMPA) & $94(78 \%)$ \\
\hline Norethisterone enanthate (NET-EN) & $27(22 \%)$ \\
\hline Hormonal implant & $50(23 \%)$ \\
\hline Sterilization & $5(2 \%)$ \\
\hline \multicolumn{2}{|l|}{ Age, years } \\
\hline Median (range) & $26(18-42)[213]$ \\
\hline
\end{tabular}

other menses-related issues, such as weight gain. Some participants attributed these side effects to their contraceptives, and they reported their issues to staff members or requested to change their method. Despite high retention in the trial, the side effects that participants experienced led them to consider discontinuation from ASPIRE.

I had a problem with the prevention. I was a person who did not like to use prevention. Now that I was in the study and using the ring, it was a requirement to use prevention. I ended up using it because it was a requirement that while I'm here I have to use it. When I started using it, it did not treat me well. I did not know whether I should quit the study or what because I menstruated for a long time. [Johannesburg, South Africa]

Furthermore, participants experienced side effects that may have been caused by contraceptives but were attributed to the study ring. Several women reported removing their rings after experiencing such side effects and choosing not to reinsert before their next study visit. When participants brought these issues to the attention of staff during clinic visits, staff often explained the side effects were caused by contraceptives, but some women remained skeptical.

For me when we had just started using the ring, I started using the IUCD as well. I bled so much because I spent three months bleeding. And whenever I would lift anything heavy, I would feel pain in the lower abdomen. I got scared and thought that maybe it is the ring that had caused that bleeding. I wondered what I could do but I never removed the ring. But almost every week I would come to the study clinic and tell the health workers that I was feeling a lot of pain and was bleeding. I asked them if it was the ring causing that, but they would tell me it is not the ring but the IUCD for family planning... [Kampala, Uganda]

Even when side effects were not experienced directly by participants, possible side effects from contraceptives were still a concern. Contraceptives' impact on fertility was a particular concern. Participants expressed fears that the contraceptive they used during the study would make them infertile or make conception difficult later on, especially if the woman had not yet had children.

People tell me if you are on contraception and you've never had a baby before it becomes complicated to have a baby in the future. Even elders say if you are [on] contraception without having borne a child then you won't have babies at all" [Durban, South Africa] 
In some cases, fears about contraception and fertility were based in misunderstanding of the reversibility of contraceptive methods.

It troubled me because... some said Depo is a problem in that when you want to get pregnant it will be a challenge and you can spend even 4 or 5 years without getting pregnant while trying to get pregnant. [Harare, Zimbabwe]

Participants further indicated that these fears could be exacerbated by the knowledge that two distinct products were in use simultaneously.

You might always be thinking about having two things inside your body; it makes you be worried all the time.... You might be worried that having those two things might cause you some problems. [Kampala, Uganda]

\section{Discussion}

Participant narratives revealed that negative side effects caused by or perceived to be caused by contraceptives could be a barrier to ring adherence. Changes in bleeding patterns, weight gain, and other effects led them to consider discontinuing study participation. Even when such effects were not experienced personally, fear of side effects generated by listening to other participants sometimes led women to question their own continued use of HIV preventative products $[5,8]$. Future participants in clinical trials or users of new HIV prevention methods outside the study environment could be prone to discontinuing HIV prevention measures if they associate known contraceptive side effects with the prevention measure. This may be of particular importance if they start using the two products simultaneously.

Actual and perceived contraceptive side effects influencing acceptance could also pose a barrier to consistent use of multipurpose technologies (MPTs) that are currently under investigation for pregnancy and HIV prevention. In ASPIRE, women cited irregular menses, abdominal pain, and other side effects as a barrier to their continued ring use, in a context where the flexibility of switching contraceptive methods was readily available. In the full study, $34 \%$ of participants had switched contraceptive methods at 12 months [5]. Because MPTs inherently link pregnancy and HIV prevention into a single product, nonadherence due to side effects for one indication would directly impact adherence to the other. Detailed counselling for these side effects could reduce discontinuation of MPT use.

Women indicated a concern that the ring or contraceptive might continue to act years after discontinuation or cause permanent damage to their fertility. This sentiment echoes results from other studies $[9,10]$. Sub-Saharan Africa is a setting in which infertility can have far-reaching effects on women's social, economic, and physical health [11]. Respectively, testing and roll-out of HIV prevention methods like the dapivirine ring or future MPT should pre-emptively address the importance of fertility to users and the community.

Use of a reliable form of contraception among participants in this trial was a requirement because of the investigational nature of dapivirine, and unknown effect of the ring during pregnancy. The Microbicide Trials Network has two trials in development to evaluate the safety of the dapivirine ring among pregnant (MTN-042) and breastfeeding (MTN-043) women [12]. Oral PrEP use in pregnancy has been supported in some HIV endemic settings and is believed to be safe [13]. Preliminary analysis of pregnancy and infant outcomes in the Phase III dapivirine ring trials indicates that the ring is not associated with adverse effects in early pregnancy [14]. However, until more definitive research results are released, the challenges that contraceptives pose when evaluating HIV prevention tools will need to be addressed for concurrent use of HIV PrEP technologies and contraceptives.

\section{Limitations}

The topic of contraception challenges was not a key objective of the ASPIRE study and was not systematically queried. However, the interview guides and codes did allow for a targeted secondary analysis of themes surrounding contraception, reproductive health, and associated barriers to ring use. The sample size of interview passages also limits the conclusiveness of thematic differences seen between sites, so site comparisons have not been explored.

Furthermore, many women in the study started or switched contraceptive methods upon joining the study [15]. In a typical environment, women considering using the ring may have an established contraceptive routine already. As a result, they could be less likely to attribute familiar contraceptive side effects to the ring.

\section{Conclusion}

Women's attitudes toward and experience with contraceptives can impact their willingness to concurrently adhere to a novel HIV prevention product like the dapivirine ring. In order to optimize the potential of both prevention products, researchers should pre-emptively address concerns about contraceptive impact on fertility, provide reassurance that contraceptives will be reliably reversible, and counsel women about expected side effects of contraceptives versus the ring. The real-world success of the dapivirine ring could be significantly 
impacted by how concerns about concurrent contraceptive products are addressed.

\author{
Abbreviations \\ FGD: Focus group discussions; MPTs: Multipurpose technologies; PUEV: \\ Product use end visit; IDI: Single in-depth interviews; SIDI: Serial in-depth \\ interviews.
}

\section{Supplementary Information}

The online version contains supplementary material available at https://doi. org/10.1186/s12905-021-01321-5.

Additional file 1. In-depth interview topic guide.

Additional file 2. Focus group discussion topic guide.

\section{Acknowledgements}

The authors express their gratitude to the ASPIRE participants. They further thank the study sites and site investigators of record. Study Team Leadership: Jared Baeten, University of Washington (Protocol Chair); Thesla Palanee-Phillips, Wits Reproductive Health and HIV Institute (Protocol Co-chair); Elizabeth Brown, Fred Hutchinson Cancer Research Center (Protocol Statistician); Lydia Soto-Torres, US National Institute of Allergy and Infectious Diseases (Medical Officer); Katie Schwartz, FHI 360 (Clinical Research Manager). Study sites and site Investigators of Record: Malawi, Blantyre site (Johns Hopkins University, Queen Elizabeth Hospital): Bonus Makanani; Malawi, Lilongwe site (University of North Carolina, Chapel Hill): Francis Martinson; South Africa, Cape Town site (University of Cape Town): Linda-Gail Bekker; South Africa, Durban Botha's Hill, Chatsworth, Isipingo, Tongaat, Umkomaas, Verulam sites (South African Medical Research Council): Vaneshree Govender, Samantha Siva, Zakir Gaffoor, Logashvari Naidoo, Arendevi Pather, and Nitesha Jeenarain; South Africa, Durban, eThekwini site (Center for the AIDS Programme for Research in South Africa): Gonasagrie Nair; South Africa, Johannesburg site (Wits RHI): Thesla Palanee-Phillips; Uganda, Kampala site (John Hopkins University, Makerere University): Flavia Matovu; Zimbabwe, Chitungwiza, Seke South and Zengeza sites (University of Zimbabwe, University of California San Francisco): Nyaradzo Mgodi; Zimbabwe, Harare, Spilhaus site (University of Zimbabwe, University of California San Francisco): Felix Mhlanga. Data management was provided by The Statistical Center for HIV/AIDS Research \& Prevention (Fred Hutchinson Cancer Research Center, Seattle, WA) and site laboratory oversight was provided by the Microbicide Trials Network Laboratory Center (Pittsburgh, PA). The MTN-020/ASPIRE study was designed and implemented by the Microbicide Trials Network (MTN). The MTN is funded by the National Institute of Allergy and Infectious Diseases (UM1AI068633, UM1AI068615 and UM1Al106707), with cofounding from the Eunice Kennedy Shriver National Institute of Child Health and Human Development and the National Institute of Mental Health, all components of the US National Institutes of Health. The content is solely the responsibility of the authors and does not necessarily represent the official views of the National Institutes of Health. The vaginal rings used in this study were developed and supplied by the International Partnership for Microbicides. The authors further thank Dr. Dorothy Holland for her mentorship.

\section{Authors' contributions}

$J L, F K, T P P, K B, F M, B K, J B, A K, S H$, and EM contributed to the interpretation of the data and reviewed and edited the manuscript. JL and ETM led the writing and development of the manuscript. FK, FM, TPP were involved with site implementation of the study protocol. AK oversaw study implementation procedures, data collection and study management. SH, JB, KB and TPP designed the study and provided scientific oversight to the design of the study protocol, data collection and procedures. SH, JB, BK, TPP, FK and FM were members of the ASPIRE study Contraceptive Action Team. JL led the data analyses. All authors read and approved the final manuscript.

\section{Funding}

The MTN-020/ASPIRE study was designed and implemented by the Microbicide Trials Network (MTN). The MTN is funded by the National Institute of
Allergy and Infectious Diseases (UM1 Al068633, UM1 Al068615, UM1Al106707), with co-funding from the Eunice Kennedy Shriver National Institute of Child Health and Human Development and the National Institute of Mental Health, all components of the U.S. National Institutes of Health. The content is solely the responsibility of the authors and does not necessarily represent the official views of the National Institutes of Health. The vaginal rings used in this study were developed and supplied by the International Partnership for Microbicides (IPM).

\section{Availability of data and materials}

The datasets generated and/or analyzed during are not publicly available due to the need to maintain participant confidentiality.

\section{Declarations}

\section{Ethics approval and consent to participate}

The study was approved by the following Institutional Review Boards/Ethics Committees and Drug Regulatory Authorities: Prevention Sciences Research Committee of the US National Institute of Allergy and Infectious Diseases; US Food and Drug Administration; National Health Sciences Research Committee of Malawi; University of North Carolina at Chapel Hill Institutional Review Board; Pharmacy, Medicines and Poisons Board of Malawi; University of Cape Town: Human Research Ethics Committee; South African Medical Research Council Ethics Committee; Human Research Ethics Committee: (Medical), University of Witwatersrand, Johannesburg; Medicines Control Council of South Africa; Joint Clinical Research Centre Institutional Review Board; Uganda National Council for Science and Technology; Johns Hopkins University School of Medicine Institutional Review Board; National Drug Authority of Uganda; Medical Research Council of Zimbabwe; Committee on Human Research, University of California-San Francisco; Joint Parirenyatwa Hospital and College of Health Sciences Research Ethics; Research Council of Zimbabwe; Medicines Control Authority of Zimbabwe. All participants provided written informed consent.

\section{Consent for publication}

Not applicable.

\section{Competing interests}

The authors declare that they have no competing interests.

\section{Author details}

${ }^{1}$ Women's Global Health Imperative, RTI International, San Francisco, CA, USA. ${ }^{2}$ Makerere University-Johns Hopkins University Research Unit, Kampala, Uganda. ${ }^{3}$ Wits Reproductive Health and HIV Institute (Wits RHI), Hillbrow, South Africa. ${ }^{4}$ Department of Obstetrics, Gynecology and Reproductive Sciences, University of Pittsburgh, Pittsburgh, PA, USA. ${ }^{5}$ UZ-UCSF Collaborative Research Programme, Harare, Zimbabwe. ${ }^{6}$ Departments of Global Health, Medicine, and Epidemiology, University of Washington, Seattle, WA, USA. ${ }^{7}$ Magee-Womens Research Institute, Pittsburgh, PA, USA.

Received: 18 September 2020 Accepted: 19 April 2021 Published online: 23 April 2021

\section{References}

1. HIV/AIDS JUNPo. Global report: UNAIDS report on the global AIDS epidemic: 2013: UNAIDS; 2013.

2. Baeten JM, Palanee-Phillips T, Brown ER, Schwartz K, Soto-Torres LE, Govender V, et al. Use of a Vaginal Ring Containing Dapivirine for HIV-1 Prevention in Women. The New England Journal of Medicine. 2016.

3. Nel A, van Niekerk N, Kapiga S, Bekker L-G, Gama C, Gill K, et al. Safety and efficacy of a dapivirine vaginal ring for HIV prevention in women. N Engl J Med. 2016:375(22):2133-43.

4. Baeten J. High adherence and sustained impact on HIV-1 incidence: final results of an open-label extension trial of the dapivirine vaginal ring. July 2019. http://programme.ias2019.org/People/PeopleDetailStandalone/ 3329. PowerPoint Presentation. 
5. Chitukuta $M$, et al. Negative rumours about a vaginal ring for HIV-1 prevention in sub-Saharan Africa. Cult Health Sex. 2019. https://doi.org/ 10.1080/13691058.2018.1552989.

6. Montgomery ET, van der Straten A, Chitukuta M, Reddy K, Woeber K, Atujuna $M$, et al. Acceptability and use of a dapivirine vaginal ring in a phase III trial. AIDS. 2017;31(8):1159-67.

7. Guest G, MacQueen KM, Namey EE. Applied thematic analysis. Los Angeles: Sage; 2012.

8. van der Straten A, Montgomery ET, Musara P, Etima J, Naidoo S, Laborde $\mathrm{N}$, et al. Disclosure of pharmacokinetic drug results to understand nonadherence. AIDS. 2015;29(16):2161-71.

9. Woodsong C, Holt JD. Acceptability and preferences for vaginal dosage forms intended for prevention of HIV or HIV and pregnancy. Adv Drug Deliv Rev. 2015;92:146-54.

10. Woodsong C, Musara P, Chandipwisa A, Montgomery E, Alleman $\mathrm{P}$, Chirenje $\mathrm{M}$, et al. Interest in multipurpose prevention of HIV and pregnancy: perspectives of women, men, health professionals and community stakeholders in two vaginal gel studies in southern Africa. BJOG. 2014;121(Suppl 5):45-52.

11. Brady M. Preventing sexually transmitted infections and unintended pregnancy, and safeguarding fertility: triple protection needs of young women. Reprod Health Matters. 2003;11(22):134-41.
12. A time to deliver: preventing HIV in pregnant and breastfeeding women. Microbicide Trials Network, 17 Oct. 2018, http://mtnstopshiv.org/news/ time-deliver-preventing-hiv-pregnant-and-breastfeeding-women.

13. WHO technical brief: preventing HIV during pregnancy and breastfeeding in the context of pre-exposure prophylaxis (PrEP). Geneva: World Health Organization; 2017. Licence: CC BY-NC-SA 3.0 IGO

14. Makanani $B$, et al. Pregnancy and infant outcomes among women using the dapivirine vaginal ring in early pregnancy. JAIDS. 2018;79(5):566-72. https://doi.org/10.1097/qai.0000000000001861.

15. Chappell CA, et al. Contraceptive method switching among women living in sub-Saharan Africa participating in an HIV-1 prevention trial: a prospective cohort study. Contraception. 2019;100(3):214-8. https://doi. org/10.1016/j.contraception.2019.06.006.

\section{Publisher's Note}

Springer Nature remains neutral with regard to jurisdictional claims in published maps and institutional affiliations.
Ready to submit your research? Choose BMC and benefit from:

- fast, convenient online submission

- thorough peer review by experienced researchers in your field

- rapid publication on acceptance

- support for research data, including large and complex data types

- gold Open Access which fosters wider collaboration and increased citations

- maximum visibility for your research: over $100 \mathrm{M}$ website views per year

At $\mathrm{BMC}$, research is always in progress.

Learn more biomedcentral.com/submissions 